\section{A avaliação de programas e serviços de saúde no Brasil enquanto espaço de saberes e práticas}

\author{
The evaluation of health programs and services in \\ Brazil as a space for knowledge and practice
}

\author{
La evaluación de los programas y servicios de \\ salud en Brasil como un espacio de \\ conocimiento y práctica
}

\begin{abstract}
${ }_{1}$ Departamento de Políticas Públicas e Saúde Coletiva, Universidade Federal de São Paulo, Santos, Brasil.

2 Instituto de Saúde Coletiva, Universidade Federal da Bahia, Salvador, Brasil.

Correspondência

J. P. Furtado

Departamento de Políticas Públicas e Saúde Coletiva, Universidade Federal de São Paulo.

RuaSilva Jardim 136, Santos, SP 11015-020, Brasil.

juarezpfurtado@hotmail.com
\end{abstract}

\begin{abstract}
This social and historical study drew on Bourdieu's genetic sociology to analyze the establishment and development of a specialized space for the production of knowledge and practices in health evaluation in Brazil. The study analyzed the trajectories of 28 researchers and policymakers and the historical conditions that allowed establishing this space, using in-depth interviews, document analysis, and a literature review. The resulting material was analyzed according to Bourdieu's concepts of field, habitus, and capital. The results point to the constitution of a sub-space for evaluation within Public Health, resulting from interaction between actors from the administrative and scientific fields, respectively, represented by management institutions in the Brazilian Unified National Health System at its various levels and research groups affiliated with public universities. No common habitus was found between the interviewees and the inherent issues and disputes in this sub-space.
\end{abstract}

Program Evaluation; Health Evaluation; Sociology
Juarez Pereira Furtado 1

Ligia Maria Vieira-da-Silva ${ }^{2}$

\section{Resumo}

Com o objetivo de analisar a constituição e o desenvolvimento de um espaço social especializado na produção de saberes e práticas sobre a avaliação em saúde no Brasil, foi realizado um estudo sócio-histórico apoiado na sociologia genética de Bourdieu. Para isso, foram analisadas as trajetórias de 28 agentes selecionados entre pesquisadores e gestores, bem como as condições históricas de possibilidade de constituição do espaço, por meio de entrevistas em profundidade, análise de documentos e revisão bibliográfica. O material gerado foi analisado à luz dos conceitos de campo, habitus e capital propostos por Bourdieu. Os resultados apontam para a constituição de um subespaço da avaliação no interior da Saúde Coletiva, resultado da interação entre agentes dos campos burocrático e científico, respectivamente representados pelas instituições de gestão do Sistema Único de Saúde (SUS) em seus vários níveis e grupos de pesquisa inseridos em universidades públicas. Não foi identificado habitus comum entre os agentes entrevistados $e$ as questões e disputas inerentes a esse subespaço.

Avaliação de Programas e Projetos de Saúde; Avaliação em Saúde; Sociologia 


\section{Introdução}

No Brasil, a avaliação em saúde constituiu-se como objeto de interesse em vários momentos históricos, seja no interior da Saúde Pública institucionalizada 1 , seja nos momentos que antecederam imediatamente a implementação do Sistema Único de Saúde (SUS) 2. O estímulo à utilização de práticas avaliativas pela Organização Mundial da Saúde (OMS) a partir da declaração de Alma-Ata encontrou eco tardiamente no país devido a dois fatores interdependentes: por um lado, as características do Estado autoritário, vigente no início dos anos 1980, avesso a submeter suas incipientes políticas sociais à avaliação ou a qualquer outro tipo de análise 3 , e, por outro lado, a sociedade brasileira estava longe de reivindicar responsabilidade e transparência nas políticas públicas de seus programas e serviços 4 . Na contracorrente, no final dos anos 1980 e início dos 1990, contribuíram, para o ingresso da temática da avaliação na agenda sanitária brasileira, as políticas de valorização do planejamento em saúde 5 e as políticas voltadas para a unificação e descentralização do sistema de saúde, como as Ações Integradas de Saúde (AIS) e os Sistemas Unificados e Descentralizados de Saúde (SUDS) 6 . Essa contínua expansão e relativa autonomização do espaço da avaliação em saúde faz emergir questões sobre a sua constituição no interior do setor Saúde no Brasil. Afinal, como e por que determinados agentes e instituições intervieram nesse processo? Como e por que foi estruturado o espaço da avaliação em saúde no Brasil, configurando o perfil atual?

Quando se pretende localizar a evolução do pensamento e das práticas da avaliação de programas e serviços no tempo, o histórico proposto por Guba \& Lincoln 7, no final dos anos 1980, é recorrente na literatura especializada. É frequente encontrar, em textos nacionais ou recentemente traduzidos, referência à metáfora das gerações de avaliação, criada pelos dois autores 7 . A partir de críticas às três gerações anteriores, caracterizadas, respectivamente, pela mensuração, descrição e julgamento, os autores 7 formularam uma nova proposta, na qual a ênfase estaria no caráter construtivista da avaliação por meio da inclusão dos agentes sociais envolvidos com a intervenção em todos os momentos da avaliação e na utilização de técnicas qualitativas de investigação, configurando a chamada "avaliação de quarta geração". Essa formulação contribuiu para o debate na área ao introduzir a importância das abordagens qualitativas e participativas para a avaliação. No entanto, a periodização proposta 7 configura-se como um artifício ao pressupor homogeneidade em cada uma das fases ou gera- ções e desconsidera o papel das instituições e dos agentes individuais nesse percurso.

Outras abordagens do desenvolvimento histórico da avaliação nos Estados Unidos apresentam limitações ao utilizarem, como eixo de análise, polarizações restritas a aspectos metodológi$\cos 8$, equivalendo o percurso histórico a estágios de desenvolvimento teórico e seus respectivos autores 9 ou se atendo aos aspectos factuais de seu desenvolvimento ${ }^{10}$. São também frequentes a caracterização da avaliação com base em distintas tipologias e modelos, agrupando autores, práticas e referenciais, porém desarticulados de referencial teórico explicativo.

A avaliação tem sido ainda analisada sob as perspectivas de disciplina acadêmica e/ou âmbito de práticas, sendo raras as iniciativas que procuram abordá-la como espaço social ou como campo. Exceção feita a dois números especiais de diferentes revistas francesas, respectivamente de Sociologia e Filosofia que, em dezenas de artigos, abordam questões ideológicas, domínios e perspectivas críticas relativas às concepções e práticas da avaliação em diferentes setores 11,12 .

Se insuficientes para a compreensão da origem e desenvolvimento da avaliação no plano internacional, as abordagens aqui apresentadas tornam-se ainda mais limitadas quando aplicadas alhures, como no caso brasileiro. Com o objetivo de investigar a constituição do espaço da avaliação em saúde no Brasil, foi realizado estudo apoiado na sociologia genética de Pierre Bourdieu, com o propósito de integrar as tradições que preconizam a objetivação metodológica com aquelas que privilegiam a subjetividade dos agentes na produção de suas representações, crenças e orientações práticas. Especialmente, no caso de Bourdieu, o modelo de relações proposto entre as noções de habitus e campo possibilita reintroduzir os agentes e suas ações singulares, estabelecendo relações com as estruturas sociais.

Considera-se que, tal como a Saúde Coletiva na qual está inserida, a avaliação em saúde constituiu-se em espaço de saberes e práticas, configurando um microcosmo social em busca de autonomia em relação às subáreas que compõem a Saúde Coletiva. Essa noção de espaço particularizado e objetivamente estruturado de relações, composto por agentes portadores de recursos materiais e simbólicos desiguais, mas que ainda assim compartilham questões, regras e crenças (illusio) no "jogo" que se pratica no interior desse mesmo espaço, perseguindo fins específicos, pode corresponder numa primeira aproximação ao conceito de campo proposto por Bourdieu 13 . Esse autor considera os diferentes campos como 
resultantes da diferenciação do mundo social em redes de relações entre agentes, com habitus e illusio comuns, detentores de questões e objetos de interesse específicos. A estrutura dos campos é influenciada pelas lutas e disputas entre agentes posicionados segundo suas diversas espécies de capital 13. Capital é aqui compreendido para além de seu sentido estritamente econômico, agregando bens de ordem simbólica e da rede de relações sociais ${ }^{14}$. Será utilizada, para a avaliação em saúde, a noção de espaço social no sentido de um âmbito de relações que não apresenta todas as características de um campo consolidado 15 .

\section{Metodologia}

Foi realizado estudo sócio-histórico sobre a constituição e o desenvolvimento do espaço da avaliação em saúde no Brasil no período compreendido entre 1990, ano da Lei Orgânica da Saúde que instituiu o SUS, e a criação do Grupo Técnico de Monitoramento e Avaliação de Programas e Políticas de Saúde da Associação Brasileira de Saúde Coletiva (GT-Avaliação da Abrasco), em 2006, durante o VIII Congresso Brasileiro de Saúde Coletiva, no Rio de Janeiro, Brasil.

Para análise das trajetórias dos agentes, bem como para a recuperação dos principais fatos relacionados com a constituição do espaço da avaliação, foram utilizadas fontes primárias, oriundas de 28 entrevistados (que serão aqui designados pela inicial "E”, seguida de um número segundo a ordem cronológica das entrevistas), todas conduzidas pelo pesquisador principal no período compreendido entre o segundo semestre de 2012 e primeiro semestre de 2013. Os agentes entrevistados que compuseram a amostra intencional preencheram, ao menos, dois dos seguintes critérios: vinculação ao GT-Avaliação da Abrasco; produção escrita e indexada relevante sobre a temática da avaliação em saúde; integrar grupo de estudo de avaliação em saúde inserido em diretório específico do Conselho Nacional de Desenvolvimento Científico e Tecnológico (CNPq); ocupação de cargos na administração federal em saúde nos quais a avaliação constitui objeto central. Eles eram oriundos de diversas profissões de saúde ou das Ciências Sociais, na sua maioria docentes vinculados a universidades, mas, também, gestores e técnicos vinculados aos serviços de saúde (Tabela 1). O local das entrevistas foi definido pelos entrevistados, tendo ocorrido em suas salas de trabalho na maioria das vezes. As análises de iniciativas relativas ao SUS ficaram circunscritas ao Ministério da Saúde, dada as limitações para uma abordagem dos níveis estaduais e municipais que compõem esse sistema e o caráter indutor que a administração federal cumpre diante dos demais.

Foram analisadas as trajetórias (profissional, social e política) dos diversos agentes, bem como as posições atualmente ocupadas no interior do espaço da avaliação. Os campos e espaços sociais são redes de relações entre agentes, relações essas que podem ser de dominação, homologia ou subordinação. Dependendo do volume do capital global acumulado, os agentes situam-se em posições dominantes (membros de comissões avaliadoras, dirigentes institucionais, dirigentes de associações) ou em posições dominadas (recém-ingressos no campo, em início de carreira etc.) $13,14,15$.

A trajetória social foi analisada por meio da ocupação dos pais e avós; a trajetória política e profissional, por meio da formação (títulos), cargos ocupados e tomadas de posição em momentos específicos pelos entrevistados. A posição de cada entrevistado no espaço da avaliação - se no polo dominado ou dominante - foi definida por meio da análise da composição e do volume das diversas espécies de capital apresentados por cada um dos agentes: capital científico, político e burocrático que foram classificados em pequeno $(\mathrm{P})$, médio $(\mathrm{M})$, alto $(\mathrm{A})$ e muito alto (AA). Os critérios utilizados e classificação dos capitais estão detalhados nas Tabelas 2 e 3, respectivamente. $\mathrm{O}$ capital simbólico que corresponde ao reconhecimento dos agentes pelos seus pares foi aferido a partir de referências espontaneamente feitas pelos entrevistados. Foram ainda analisadas as condições de possibilidade históricas apoiadas em fontes bibliográficas e documentais.

Os conteúdos apreendidos por meio das entrevistas foram complementados com livros e artigos indexados de autoria dos entrevistados, manuais institucionais do Ministério da Saúde referentes à avaliação em saúde, currículos dos entrevistados constantes na plataforma Lattes do CNPq, documentos da Associação Brasileira da Pós-graduação em Saúde Coletiva (Abrasco) e relatórios do GT-Avaliação (arquivos pessoais dos entrevistados). Os livros e artigos foram selecionados a partir do Currículo Lattes dos entrevistados.

Para análise do campo cientifico, além das trajetórias dos agentes, foi realizada revisão dos artigos publicados no período compreendido entre 1990 e 2006, com as palavras-chave "avaliação em saúde"; "avaliação de programas em saúde"; "avaliação de programas e projetos de saúde" na base SciELO, nos índices de título e assunto. Os artigos localizados foram analisados quanto ao número e objeto das publicações. O objetivo dessa revisão parcial foi verificar a evolução quantitativa da produção nacional sobre avalia- 
Tabela 1

Perfil dos entrevistados.

\begin{tabular}{|c|c|c|c|c|c|c|}
\hline Entrevistado & Sexo & Graduação & $\begin{array}{c}\text { Ano da } \\
\text { graduação }\end{array}$ & $\begin{array}{l}\text { Formação em } \\
\text { Saúde Coletiva }\end{array}$ & Vinculação institucional & Atuação profissional \\
\hline E1 & $\mathrm{M}$ & Medicina & 1971 & ES, MT, DT & Universidade & Ensino \& pesquisa \\
\hline E2 & $\mathrm{F}$ & Medicina & 1971 & MT, DT, PD & Universidade & Ensino \& pesquisa \\
\hline E3 & $\mathrm{F}$ & Medicina & 1983 & ES, MT, DT & Universidade & Pesquisa \\
\hline E4 & $\mathrm{F}$ & Medicina & 1974 & ES, MT, DT, PD & Universidade & Ensino \& pesquisa \\
\hline E5 & $\mathrm{F}$ & Medicina & 1975 & MT, PD & Universidade & Pesquisa \\
\hline E6 & $\mathrm{F}$ & Psicologia & 1993 & ES, MT, DT & Instituição de pesquisa & Pesquisa \\
\hline E7 & $\mathrm{F}$ & Medicina & 1979 & R, MT, DT, PD & Universidade & Gestão \\
\hline E8 & $\mathrm{F}$ & Medicina & 1976 & ES, MT, DT & Universidade & Ensino \& pesquisa \\
\hline E9 & $\mathrm{F}$ & Medicina & 1977 & MT, DT & Universidade & Pesquisa \\
\hline E10 & $\mathrm{F}$ & Nutrição & 1986 & ES, DT & $\begin{array}{l}\text { Instituição de pesquisa e } \\
\text { assistência }\end{array}$ & Pesquisa \\
\hline E11 & $\mathrm{M}$ & Medicina & 1984 & $\mathrm{R}, \mathrm{MT}, \mathrm{DT}$ & $\begin{array}{c}\text { Secretaria de Estado da } \\
\text { Saúde }\end{array}$ & Gestão \\
\hline E12 & $\mathrm{F}$ & Medicina & 1993 & MT, DT & $\begin{array}{l}\text { Instituição de pesquisa e } \\
\text { assistência }\end{array}$ & Ensino \& pesquisa \\
\hline E13 & $\mathrm{F}$ & Medicina & 1986 & $\mathrm{ES}, \mathrm{MT}, \mathrm{DT}$ & Universidade & Ensino \& pesquisa \\
\hline E14 & M & Medicina & 1979 & MT, PD & Universidade & Ensino \& pesquisa \\
\hline E15 & M & Medicina & 2003 & ES & Ministério da Saúde & Gestão federal \\
\hline E16 & M & Fisioterapia & 1988 & $\mathrm{R}$ & Ministério da Saúde & Gestão federal \\
\hline E17 & M & Medicina & 1985 & $\mathrm{R}, \mathrm{MT}$ & Ministério da Saúde & Gestão federal \\
\hline E18 & $\mathrm{F}$ & Odontóloga & 1981 & $\mathrm{ES}, \mathrm{MT}$ & Universidade & Ensino \& pesquisa \\
\hline E19 & $\mathrm{F}$ & Enfermagem & 1986 & ES & Universidade & Ensino \& pesquisa \\
\hline E20 & $\mathrm{F}$ & Sociologia & 1990 & MT, DT & Universidade & Pesquisa \\
\hline E21 & $\mathrm{F}$ & Medicina & 1983 & ES, MT, DT & Universidade & Ensino \& pesquisa \\
\hline E22 & M & Medicina & 1970 & $\mathrm{MT}$ & Instituto de pesquisa & Pesquisa \\
\hline E23 & M & Medicina & 1981 & ES, MT, DT & Universidade & Ensino \& pesquisa \\
\hline E24 & M & $\begin{array}{l}\text { Administração de } \\
\text { Empresas }\end{array}$ & 1975 & PD & Universidade & Ensino \& pesquisa \\
\hline E25 & $\mathrm{F}$ & Enfermagem & 1992 & MT, DT & Universidade & Ensino \& pesquisa \\
\hline E26 & M & Ciências Econômicas & 1973 & $\mathrm{MT}$ & Universidade & Gestão municipal \\
\hline E27 & M & Medicina & 1975 & & Ministério da Saúde & Gestão federal \\
\hline E28 & $\mathrm{F}$ & Medicina & 1979 & ES, MT, DT, PD & Universidade & Ensino \& pesquisa \\
\hline
\end{tabular}

DT: doutorado; E: entrevistado; ES: especialização; F: feminino; M: masculino; MT: mestrado; PD: pós-doutorado; R: residência.

ção em saúde ao longo do período analisado. O presente estudo limitou-se à análise do espaço da avaliação de políticas e programas de saúde. Outros subespaços, a exemplo daquele que toma por objeto a avaliação de tecnologias de saúde, não foram incluídos, tendo, em vista, a delimitação do escopo da investigação. Essa pesquisa foi aprovada pelo Comitê de Ética da Universidade Federal de São Paulo sob o parecer de número $56658 / 12$.

\section{Resultados e discussão}

\section{Condições de possibilidade históricas}

A constituição do espaço social de relações entre pesquisadores, técnicos e gestores interessados em realizar avaliações de programas de saúde foi favorecido por três processos sociais concomitantes. Por um lado, a organização e estruturação do SUS no país, que resultou na formulação e implantação de políticas setoriais e globais que requeriam avaliação dos seus resultados, ao lado da própria constituição do espaço da Saúde 
Critérios para análise dos capitais científico, político e burocrático por referência ao espaço da avaliação em saúde no Brasil.

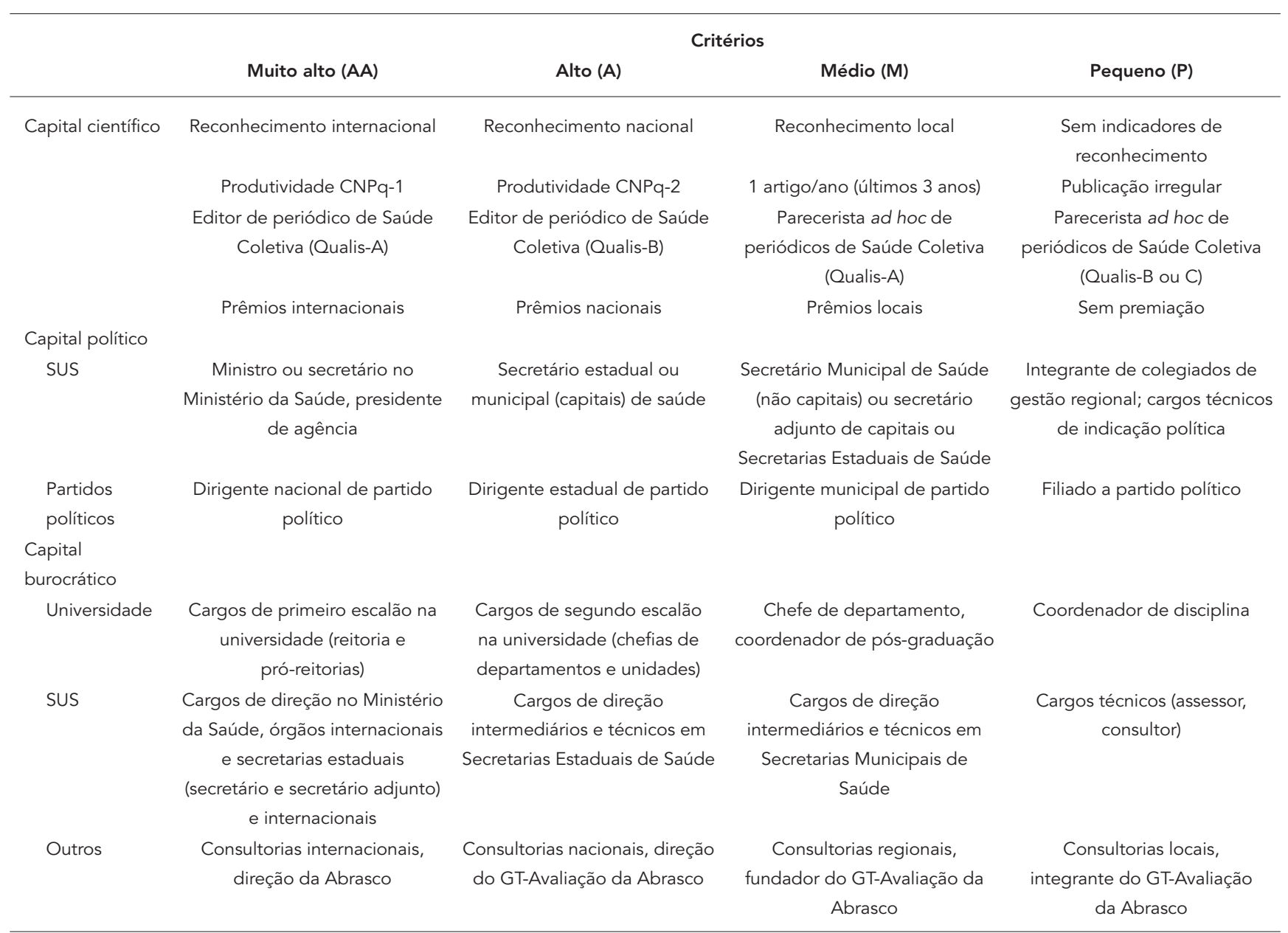

CNPq: Conselho Nacional de Desenvolvimento Científico e Tecnológico; GT-Avaliação da Abrasco: Grupo Técnico de Monitoramento e Avaliação de Programas e Políticas de Saúde da Associação Brasileira de Saúde Coletiva.

Fonte: adaptado de Vieira-da-Silva \& Pinell 36 e Bourdieu 37.

Coletiva, que aglutinou pesquisadores e gestores em torno dessa temática. Por outro lado, a indução exercida pelo financiamento de instituições internacionais expressa nas exigências de avaliação de projetos constituiu outro fator importante para a inserção de práticas avaliativas no interior do SUS, em geral, e na atenção básica, em particular 16,17. O envolvimento pessoal de alguns dos entrevistados nesse processo foi enfatizado:

"O projeto Reforsus do Banco Mundial injetou muito dinheiro nesse país, durante quase sete anos. Ele foi impulsionando a necessidade de avaliar, e eu participei pessoalmente de algumas tarefas como essa" (E1, médico, docente).

Tais exigências foram não só atendidas como incorporadas e fomentadas, por meio da busca de institucionalização da avaliação, ocor- rida anos mais tarde, no âmbito do Ministério da Saúde. Cabe destaque ao Reforsus (Reforço e Reorganização do SUS), programa governamental instituído em 1996, envolvendo repasses de 650 milhões de dólares do Banco Internacional para Reconstrução e Desenvolvimento (BIRD) e do Banco Interamericano de Desenvolvimento (BID) que teve, entre outros objetivos, o de incrementar a qualidade da assistência e da gestão 18 , incorporando o uso da avaliação tanto para liberação de financiamentos como para apreciação do impacto dos projetos. Segundo um entrevistado, que participou como consultor de um desses projetos:

“...Sem dúvida, para mim, esse movimento internacional de financiadores externos tinha como pressuposto obrigar que os financiados usassem as 
Tabela 3

\begin{tabular}{|c|c|c|c|}
\hline Agente & Científico & Burocrático & Político \\
\hline E1 & A & A & $M$ \\
\hline E2 & $\mathrm{AA}$ & $M$ & $P$ \\
\hline E3 & $M$ & $M$ & $P$ \\
\hline E4 & $M$ & $M$ & $P$ \\
\hline E5 & AA & $P$ & $P$ \\
\hline E6 & $A$ & A & $P$ \\
\hline E7 & $A$ & A & $P$ \\
\hline E8 & $A$ & $P$ & $P$ \\
\hline E9 & AA & A & $M$ \\
\hline E10 & $M$ & $P$ & $P$ \\
\hline E11 & A & $A A$ & $M$ \\
\hline E12 & $M$ & $P$ & $P$ \\
\hline E13 & A & $M$ & $P$ \\
\hline E14 & $\mathrm{AA}$ & A & $\mathrm{M}$ \\
\hline E15 & $P$ & AA & $\mathrm{M}$ \\
\hline E16 & $P$ & $M$ & $\mathrm{M}$ \\
\hline E17 & $\mathrm{P}$ & M & $\mathrm{P}$ \\
\hline E18 & $A$ & $P$ & $P$ \\
\hline E19 & AA & A & $P$ \\
\hline E20 & A & M & $\mathrm{P}$ \\
\hline E21 & $M$ & $A$ & $P$ \\
\hline E22 & $M$ & $M$ & $P$ \\
\hline E23 & $M$ & A & $\mathrm{M}$ \\
\hline E24 & $M$ & A & $P$ \\
\hline E25 & A & $P$ & $P$ \\
\hline E26 & $P$ & A & $M$ \\
\hline E27 & $P$ & A & $M$ \\
\hline E28 & $A$ & $P$ & $P$ \\
\hline
\end{tabular}

AA: muito alto; $A$ : alto; $M$ : médio; P: pequeno.

*Vide critérios.

mesmas regras dos países de origem. (...) É preciso pensar que o Banco Mundial é um dos melhores produtores de publicação na área de avaliação" (E7, médico, pesquisador).

Os financiadores sugeriram parcerias com universidades e o terceiro setor para a realização de avaliações.

"6.33 - Evaluation. The Project Management Unit would retain the services of an independent research organization, such as a university or a research oriented nongovernmental organization (NGO), to carry out an ex-post evaluation of a sample of completed projects" 16 (p. 29).

Além do desenvolvimento específico de ações avaliativas, o Reforsus possibilitou o investimento indireto na prática avaliativa por meio de ações, junto às Secretarias Estaduais de Saúde, para desenvolver a capacidade de gestão, com ênfase no planejamento e na avaliação dos projetos 19 , tendo sido previstos com essa finalidade a quantia de 545 mil dólares.

\section{No campo burocrático}

Por meio das trajetórias dos agentes, recuperadas a partir das entrevistas, percebeu-se que as interações entre agentes dos campos científico e burocrático são imbrincadas. Ainda que seja possível caracterizar alguns agentes com trajetória predominante no interior de cada um desses campos, científico ou burocrático, é o trânsito entre esses dois universos que se constitui marca fundamental do espaço da avaliação em saúde aqui investigado. Dessa forma, diversos agentes cuja inserção principal é a universidade, como docentes e pesquisadores, já ocuparam um cargo como gestores ou assessores nos diversos níveis do SUS, o que também explica a variação na composição dos capitais científico, burocrático e político (Figura 1).

A relação entre os grupos de pesquisa em avaliação e as iniciativas ministeriais a esse respeito ilustra essa interação. Os primeiros grupos de pesquisa foram organizados nos anos 90, no interior dos Departamentos de Medicina Preventiva e Social, bem como em Escolas de Saúde Pública (E4, E7, E9). Naquele período, esses grupos universitários forneceram, em parte os consultores requeridos para desenvolverem os compromissos de avaliação firmados entre o Ministério da Saúde e o BIRD e o BID. Já no início do século XXI, verificou-se maior protagonismo por parte do Ministério da Saúde. Ainda que continuando a contratar consultorias, o Ministério da Saúde passou também a conduzir processos avaliativos com pessoal próprio ou a fomentar ações avaliativas pelas universidades, por meio de editais.

Cabe um destaque ao Programa de Expansão e Consolidação da Saúde da Família (PROESF) que, com financiamento do BIRD e do governo brasileiro, apoiou, a partir de 2003, transferências de recursos financeiros, fundo a fundo, para a expansão da cobertura, qualificação e consolidação da Estratégia Saúde da Família em municípios com população superior a $100 \mathrm{mil} \mathrm{habi-}$ tantes. O PROESF apresentou três componentes centrais, sendo que dois deles diziam respeito, direta ou indiretamente, à avaliação 20. Nesse caso, as interações entre agente e estrutura são emblemáticas: a oportunidade propiciada pelo PROESF foi potencializada pelo coordenador de acompanhamento e avaliação da atenção básica, vinculado à diretoria de atenção básica do Ministério da Saúde, entre 2003 e 2006. Tratava- 
Agentes do espaço da avaliação de políticas e programas de saúde segundo o volume de capitais científico, burocrático e simbólico.

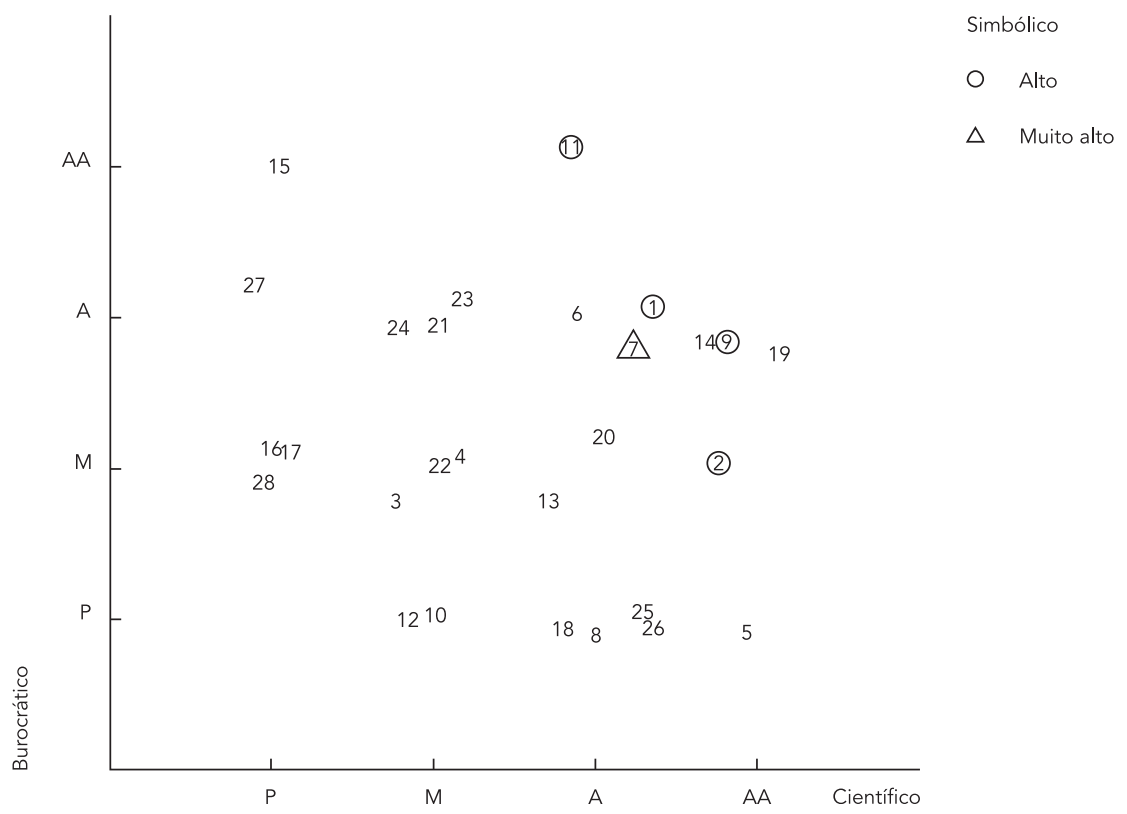

AA: muito alto; $A$ : alto; $M$ : médio; P: pequeno

se de um médico, com residência em medicina preventiva e mestrado em saúde pública, cuja formação em avaliação, iniciada em 1994, decorreu da necessidade de a instituição, a qual estava vinculado, elaborar um plano de avaliação para obter empréstimo do BID. Por essa razão, ele pode aproveitar a oportunidade que se apresentava, ou seja, um agente, com trajetória dominante no campo burocrático e sensibilizado com a temática pelo acúmulo técnico na área da avaliação, foi decisivo para a institucionalização da avaliação nas secretarias estaduais de saúde em articulação com o fomento à criação de centros colaboradores em avaliação na maioria das instituições de Ensino Superior do país 21. A relação entre o PROESF e o campo científico, representado pelas universidades brasileiras, foi profícua e se deu por meio de três vias: (a) realização de estudo de "linha de base", a partir de 2005, para estabelecer parâmetros a serem acompanhados com a implementação do PROESF em 168 municípios; (b) indução de criação de cerca de vinte centros colaboradores em avaliação para elaboração e implementação de projetos voltados à institucionalização da avaliação nas secretarias estaduais; (c) edital de pesquisa, agenciado pelo CNPq, para realização de dezenas de pesquisas avaliativas na atenção básica 22 .

Diferentemente do momento referente à primeira etapa do Reforsus, no qual o protagonismo dos financiadores denotava unilateralidade de interesses, na conjuntura de operacionalização do PROESF, a presença de agentes com formação na área, com trajetória política ligada à Reforma Sanitária, quer inseridos no governo, quer como consultores, levou à busca de consolidação da avaliação no interior da gestão do SUS (E7, E11, E26, E27). Sob a bandeira da institucionalização da avaliação, houve contraponto à pretensão dos financiadores externos em estabelecer autonomamente quais seriam os agentes realizadores e a metodologia da avaliação (E11). Os agentes do Ministério da Saúde passaram a procurar privilegiar a capacidade nacional na área.

“...Na avaliação do PROESF, (...) nós tivemos uma batalha muito grande, (...), com o Banco Mundial, que queria abrir um processo licitatório para a contratação de uma instituição única para fazer os estudos no Brasil inteiro. Então, uma das questões que a gente colocou na mesa foi o fortale- 
cimento das instituições brasileiras no campo da avaliação" (E.11, médico, gestor e pesquisador).

Essa forte interação entre agentes que ocupavam posições dominantes no campo burocrático com aqueles do campo científico gerou contribuições importantes ao desenvolvimento do espaço da avaliação de políticas e programas de saúde no Brasil, no início dos anos 2000.

\section{No campo científico}

O espaço da avaliação no interior do campo científico desenvolveu-se principalmente a partir dos anos 90, como revela o aumento da produção de artigos na primeira década do século XXI (Figura 2). Também a expansão do interesse pela temática da avaliação na academia é evidenciada pelo significativo crescimento de grupos de pesquisa de avaliação em saúde na base de diretórios do CNPq. Consulta realizada pelo GT-Avaliação da Abrasco a essa base de dados revelou a existência de 202 grupos distribuídos entre 76 diferentes instituições de Ensino Superior, sendo que dois terços desse total surgiram a partir do ano 2000, coincidindo com o aumento de publicações, como ilustrado na Figura 2. Tal crescimento encontrou paralelo nos congressos realizados pela Abrasco no período aqui considerado (1990-2006): a partir do sexto congresso, no ano 2000, há significativo incremento no número de trabalhos apresentados em comunicações coordenadas e temas livres, conforme pudemos constatar revisando a programação e os anais desses encontros.

O estímulo do Ministério da Saúde aos grupos de pesquisa de universidades públicas, por meio de contratação de consultorias e editais de pesquisa, pode explicar a simultaneidade entre a curva ascendente de publicações apresentadas na Figura 2 e as várias investigações financiadas com os recursos do PROESF para a atenção básica. Outro fator adveio da prática de gestão de sistemas e implantação de políticas e programas de saúde, gerando problemas e indagações das quais alguns agentes envolvidos nessas mesmas práticas partiram em direção ao campo científico, na busca de respostas e esclarecimentos 23,24,25. Na maioria das vezes, tratava-se de agentes inseridos na gestão de redes ou serviços do SUS, porém oriundos ou que manti-

Figura 2

Publicações sobre avaliação em saúde, segundo ano, na base SciELO (1990-2008).

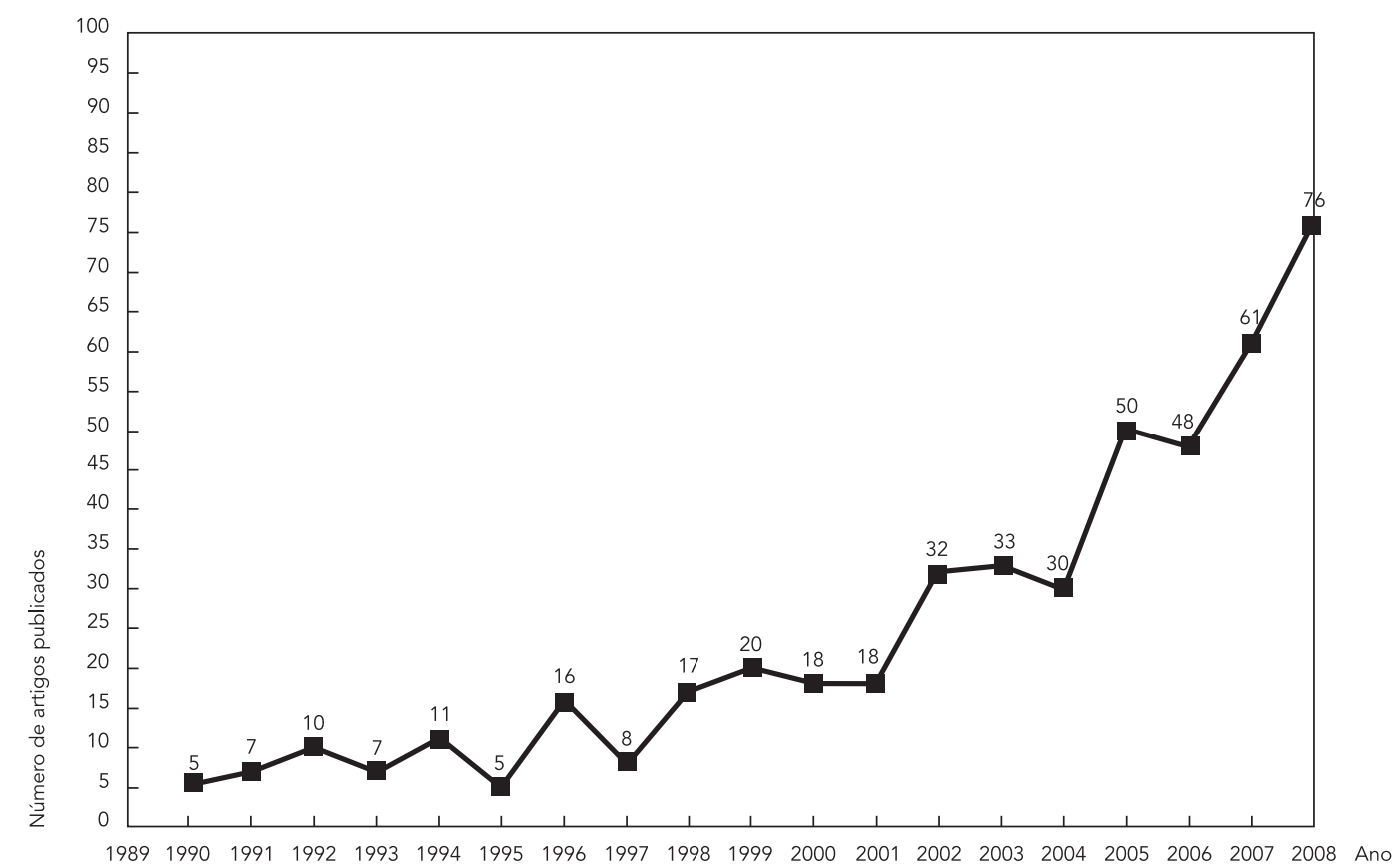

Fonte: base SciELO. 
veram vinculação paralela a grupos de pesquisa em universidades.

$\mathrm{Na}$ revisão bibliográfica realizada na base SciELO, foram localizados 835 artigos distribuídos entre vinte diferentes periódicos, incluindo números especiais dedicados ao tema, sendo que três revistas de Saúde Coletiva (Cadernos de Saúde Pública, Ciência \& Saúde Coletiva e Revista de Saúde Pública) são responsáveis por 287 artigos desse total, no período de 1990-2006 (Figura 2). Os resultados diferentes daqueles encontrados por Fernandes et al. 26, que localizaram apenas 42 artigos, podem relacionar-se com o uso de mais palavras-chave referentes à avaliação de temas específicos como a Saúde Bucal 27, a Nutrição 28 e a Atenção Básica 29.

Dois autores foram invariavelmente citados pela totalidade dos entrevistados como referencias fundamentais no desenvolvimento da avaliação em nosso país. Avedis Donabedian (19192000), médico radicado nos Estados Unidos, foi um estudioso do cuidado médico, com destaque para a noção de qualidade, sintetizada nos chamados sete pilares da qualidade 29 e com influência teórica reconhecida 2,5. Zulmira Hartz, médica, pesquisadora titular em Epidemiologia na Escola Nacional de Saúde Pública Sergio Arouca, Fundação Oswaldo Cruz (ENSP/Fiocruz), além de contribuições teóricas, articulou agentes e estabeleceu relações de impacto na constituição da avaliação como um espaço social específico.

No início dos anos 1990, alguns autores apontaram as insuficiências de processos avaliativos centrados em indicadores de qualidade 30 , havendo tentativas de aproximação entre pesquisa de qualidade e Antropologia ou mesmo a proposição do uso exclusivo de abordagens antropológicas em avaliação de serviços de saúde ${ }^{31}$. O artigo intitulado Avaliação em Saúde: Limites e Perspectivas ${ }^{32}$ pode ser considerado como a transição entre o pensamento donabediano e a avaliação de programas e serviços em saúde no Brasil, herdeira da Program Evaluation americana. O referido artigo 32 contém uma crítica à concepção de harmonia presente no referencial sistêmico usado por Donabedian 33, como na tríade estrutura-processo-resultado, desenhada para o âmbito clínico. Naquele texto 33, outras possibilidades e novas abordagens em avaliação oriundas da Antropologia, Epidemiologia ou mesmo mistas são consideradas, ainda que predomine, no artigo, a interlocução com a Epidemiologia, área hegemônica à época.

O livro Avaliação em Saúde: Dos Modelos Conceituais à Prática na Análise da Implantação de Programas, organizado por Zulmira Hartz ${ }^{34}$, publicado em 1997, constitui marco e ponto de inflexão da avaliação em saúde no Brasil em di- reção às concepções presentes em torno da chamada Program Evaluation. Um dos méritos do grupo canadense, que escreveu um dos capítulos do referido livro 34 , foi justamente o de articular as diversas propostas de origem americana à área da saúde, rompendo com a hegemonia de modelos experimentais e dicotomias, tais como estudo descritivo versus estudo analítico, avaliação formativa versus somativa, referencial quantitativo versus qualitativo, valorizando os estudos de caso. Nesse sentido, a inflexão demarcada pela publicação citada 34 é justamente a de ampliação do objeto da avaliação para além da categoria qualidade da atenção médica em senso estrito presente na obra de Donabedian 33; de extensão do leque de possibilidades metodológicas; o destaque para as análises de implantação dos programas fugindo dos modelos denominados de black box e, finalmente, o propósito em subsidiar a tomada de decisão.

Duas questões emergiram das entrevistas no que tange às relações entre o espaço da avaliação e o campo científico. A primeira delas diz respeito à diferenciação entre pesquisa avaliativa e avaliação para gestão. Nesse sentido, há diferentes visões, que correspondem a diferentes tomadas de posição, entre os entrevistados. Por um lado, situam-se aqueles que diferenciam pesquisa avaliativa (para gerar novos conhecimentos) de outros tipos de avaliação, e, de outro lado, os que identificam fronteiras cada vez mais fluidas entre gestão e avaliação. No primeiro grupo, encontram-se pesquisadores e docentes, e, no segundo, os agentes ligados à gestão ou vinculados a atividades de prestação de assessoria. A segunda questão diz respeito à utilização de técnicas quantitativas ou qualitativas em avaliação. Nesse caso, podem ser agrupadas três concepções distintas: de complementaridade entre os dois referenciais, de definição por um ou outro de acordo com o objeto avaliado e, finalmente, de crítica à hegemonia do referencial quantitativo e/ou epidemiológico. No dizer de um entrevistado:

“Agora, acho que... ainda tem uma certa hegemonia no campo avaliativo que desconhece (...) as inúmeras contribuições que a Antropologia poderia estar dando para o campo da avaliação" (E20, sociólogo, pesquisador).

E em outra perspectiva:

"É muito frágil a abordagem qualitativa para o gestor. Veja o IDSUS, quarenta indicadores totalmente quantitativos. (...) a abordagem qualitativa do sujeito (...) não tem repercussão" (E1, médico, docente). 
O GT-Avaliação Abrasco como interface dos dois campos

A criação do primeiro fórum oficial brasileiro, para a congregação de pesquisadores e demais interessados em avaliação, ocorreu durante o oitavo congresso da Abrasco, em 2006, no Rio de Janeiro, quando foi criado o GT-Avaliação. A oficina para estruturação desse grupo foi apoiada financeiramente pelo Ministério da Saúde, por meio do PROESF (E9, E11). Com o objetivo explícito de fomentar a cooperação entre universidades e a articulação entre academia e serviços de saúde, no que tange à avaliação, o GT-Avaliação estruturou suas perspectivas de ações em torno dos eixos de produção de conhecimento, formação e aplicação do conhecimento. O contexto internacional de institucionalização da avaliação, o crescimento da produção científica sobre o tema no país e as diversas iniciativas ministeriais foram justificativas utilizadas para a constituição desse grupo.

Embora a iniciativa tenha sido de agente situado no campo burocrático, dentre os trinta participantes que subscreveram a oficina de criação do GT-Avaliação, 27 eram pesquisadores ligados a universidades públicas ou institutos de pesquisa e três ao Ministério da Saúde. Naquele congresso da Abrasco, no qual foi discutido e lançado o GT-Avaliação, ocorreu o lançamento do número especial de avaliação, da revista Ciência \& Saúde Coletiva 35, cujo eixo central era a Avaliação Como Estratégia de Mudança na Atenção Básica, composto por vários artigos originados de pesquisas financiadas por editais oriundos do PROESF cujos autores participaram da reunião de criação do GT-Avaliação.

Mesmo na atualidade, o GT-Avaliação continua refletindo estreita ligação e relativa dependência do espaço da avaliação em relação ao campo burocrático, sendo o seu financiamento exclusivamente oriundo de fontes institucionais estatais. A criação do GT-Avaliação no interior da Abrasco não foi pacífica. Houve resistências oriundas de representantes tanto da Epidemiologia como das Ciências Sociais com argumentos baseados, sobretudo, no ponto de vista de que a avaliação seria transversal ao conjunto das três áreas. Posteriormente, o GT-Avaliação passou a compor a comissão de políticas daquela instituição.

\section{Os agentes do espaço da avaliação}

O fato de o interesse pela avaliação ter surgido por questões práticas ou movido por questões acadêmicas não significou a fixação dos agentes no campo que originou esse mesmo interesse. Há agentes cujo interesse surgiu a partir da gestão e que, mais tarde, fixaram-se em instituições acadêmicas e vice-versa. Há ainda casos nos quais houve equilíbrio na inserção nos dois campos considerados, ou seja, o agente ocupou cargos e cumpriu funções relevantes e/ou extensas no campo burocrático e também desenvolveu atividades de importância no campo científico.

Quando perguntados sobre a relação entre perspectivas de gestão e pesquisa, certa polarização se estabeleceu entre as opiniões dos entrevistados. Na Figura 3, o posicionamento no centro indica os agentes que realizam clara distinção entre avaliação para gestão e pesquisa avaliativa, ou seja, consideram os dois elementos como componentes da área, porém com funções e justificativas de utilização distintas. O posicionamento próximo a um dos polos corresponde ao ponto de vista dos entrevistados que definem a avaliação como uma atividade voltada para a gestão, e, no polo oposto, estão aqueles que reduzem a avaliação à pesquisa avaliativa. Finalmente, o posicionamento no exterior da figura, destacado por uma elipse, indica os agentes que consideram a avaliação um conjunto de ações de monitoramento para a gestão e pesquisa simultânea e articuladamente sem fronteiras definidas. Nesse último grupo, concentram-se, sobretudo, agentes profundamente envolvidos na gestão do SUS.

Os agentes pertencentes ao espaço da avaliação possuem uma composição variada de capitais, sendo que a situação mais frequente é aquela da composição mista de capitais burocrático e científico entre médio e alto (Figura 1). O posicionamento dos agentes em um campo está relacionado com o acúmulo de capitais valorizados naquele espaço. No caso da avaliação, o polo

Figura 3

Concepções de avaliação segundo sua vinculação com a pesquisa e gestão.

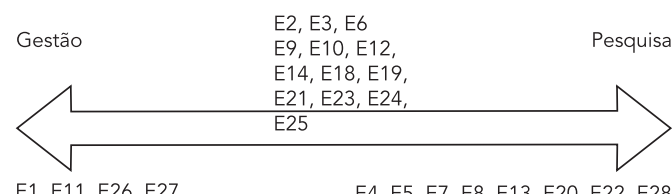

E1, E11, E26, E27

E4, E5, E7, E8, E13, E20, E22, E28

E15, E16, E17 
dominante pode ser considerado como composto pelos agentes que compuseram as coordenações do GT-Avaliação da Abrasco ou editorias de revistas de avaliação ou coordenaram programas de pós-graduação específicos, estando constituído por agentes que acumulavam capitais científico e burocrático elevados. Nesse sentido, a importância dos dois campos na constituição do espaço pode ser ilustrada pelo fato de que os agentes com maior capital simbólico são justamente aqueles que conseguiram conciliar maior capital científico e burocrático simultaneamente (Figura 1).

É significativo que nenhum dos entrevistados se identifique como avaliador ou, ao menos, como integrante do espaço "da avaliação", o que revela a situação incipiente e ambivalente em relação à constituição desse mesmo espaço. Indagados sobre como se identificavam profissionalmente, os agentes responderam explicitando sua formação na graduação ou, a menor parte, referindo sua condição de docente e pesquisador. As trajetórias híbridas, com trânsito entre os campos científico, burocrático e até mesmo político, bem como as tomadas de posição em relação às questões em jogo revelam que os habitus que estruturam o espaço da avaliação são os $h a$ bitus dos campos dominantes nas trajetórias dos agentes. Não foram identificadas convergências ou similaridades suficientes para caracterizar um habitus específico entre os agentes da avaliação considerados.

\section{Considerações finais}

A constituição do espaço da avaliação em saúde no Brasil ocorreu como produto do encontro de agentes (com trajetórias diferenciadas nos campos burocrático, político e científico) estimulados e financiados por instituições internacionais ligadas ao campo econômico, mas vinculadas à organização do SUS. Esse movimento se deu no interior do espaço da Saúde Coletiva, por sua vez um universo de saberes e práticas composto por subespaços científico, burocrático e político por onde os agentes, em boa parte, transitam 36 .

A consolidação do subespaço científico da avaliação em saúde parece caracterizar-se por progressivo distanciamento e aquisição de autonomia em relação ao Planejamento, à Epidemiologia e às Ciências Sociais, adquirindo especificidades metodológicas relativas ao objeto da pesquisa avaliativa, embora recorrendo às diversas disciplinas dessas três subáreas da Saúde Coletiva. A criação de disciplinas específicas sobre avaliação, nos programas de pós-graduação em Saúde Coletiva, e, posteriormente, a criação de mestrados profissionalizantes também voltados para essa temática são indícios do aumento de sua autonomia relativa.

Paradoxalmente, seu ganho de autonomia no interior da área de Política e Planejamento da Saúde Coletiva se fez por meio do aumento da dependência externa em relação ao Estado. Esse significativo aporte de demandas e recursos oriundos de gestores situados em diversos níveis do SUS pode ser compreendido como condição de possibilidade histórica ao efetivo desenvolvimento da pesquisa e das práticas avaliativas em saúde.

A peculiar proximidade entre os espaços de gestão e academia na avaliação em saúde faz emergir uma das disputas presentes no espaço em relação às fronteiras entre pesquisa, avaliação e gestão. A importância da avaliação para agentes do campo burocrático os leva a disputar com agentes do campo científico a definição do que é e de como se desenvolvem processos avaliativos.

Os achados do presente estudo são indicativos da existência de um processo de constituição de um espaço social formado por agentes e instituições que têm, como interesse comum, a avaliação em saúde. Contudo, a pequena autonomia relativa, a sua elevada dependência externa ao lado da ausência de identidade de seus agentes como avaliadores ou mesmo da existência de um habitus comum não permitem se falar na existência de um campo consolidado de avaliação em saúde, no sentido de Bourdieu 13 e Bourdieu \&Wacquant 15 . Esse autor, por vezes, usava o conceito de espaço social como sinônimo de campo, porém, o conceito por ele desenvolvido teoricamente foi o de campo 13. Quando um espaço de relações não possui todas as características de um campo consolidado a sua caracterização como um espaço de relações entre agentes pertencentes a campos diferentes permite uma melhor compreensão da sua natureza e significado.

O espaço da avaliação em saúde pode consolidar-se ou não como campo. A transitoriedade ou permanência desse processo relaciona-se às lutas internas ao espaço, mas, sobretudo, às suas relações com os outros campos sociais. A evolução futura das condições históricas que tornaram possível o seu surgimento, o reconhecimento que as avaliações de programas e políticas possam proporcionar aos avaliadores, bem como os usos que os resultados das avaliações possam ter por parte dos gestores irão definir ou não a sua consolidação como um campo de saberes e práticas. 


\section{Resumen}

Con el objetivo de analizar la formación y el desarrollo de un espacio social especializado en la producción de conocimientos y prácticas sobre la evaluación de la salud en Brasil se llevó a cabo un estudio sobre sociología genética, apoyado en el enfoque socio-histórico de Bourdieu. Para ello, se analizaron las trayectorias de 28 evaluadores seleccionados entre investigadores y gestores, así como las condiciones históricas de posibilidad para la creación de un espacio, a través de entrevistas en profundidad, análisis de documentos y revisión de la literatura. El material generado se analizó de acuerdo a los conceptos de campo, habitus y de capital propuestos por Bourdieu. Los resultados formaron un subespacio de evaluación en salud colectiva, resultado de la interacción entre los agentes de los campos burocráticos y científicos, representados respectivamente por las instituciones del Sistema Único de Salud (SUS) en sus diferentes niveles y grupos de investigación inscritos en las universidades públicas. No se identificó habitus comunes entre los agentes entrevistados y las cuestiones y disputas sobre el espacio.

Evaluación de Programas y Proyectos de Salud; Evaluación en Salud; Sociología

\section{Colaboradores}

J. P. Furtado realizou o trabalho de campo, concebeu a pesquisa e redigiu o artigo. L. M. Vieira-da-Silva contribuiu com a concepção do desenho da pesquisa, sua análise, revisão e redação final do artigo.

\section{Agradecimentos}

Ao Instituto de Saúde Coletiva, Universidade Federal da Bahia, pela acolhida ao nosso projeto de pós-doutorado e apoio ao longo de todo o trabalho de pesquisa empreendido. À FAPESP (2012/10550-5) e ao CNPq (150637/2012-8) pelo financiamento.

\section{Referências}

1. Ramos R. O problema da avaliação em saúde pública. Rev Saúde Pública 1974; 8:305-14.

2. Reis EJFB, Santos FP, Campos FE, Acúrcio FA, Leite MTT, Leite MLC, et al. Avaliação da qualidade dos serviços de saúde: notas bibliográficas. Cad Saúde Pública 1990; 6:50-61.

3. Ceneviva R, Farah MFS. O papel da avaliação de políticas públicas como mecanismo de controle democrático da administração pública. In: Guedes AM, Fonseca F, organizadores. O controle social da administração pública. São Paulo: Editora Unesp; 2007. p. 129-56.

4. Campos AM. Accountability: quando poderemos traduzi-la para o português? Rev Adm Pública 1990; 24:30-50.
5. Silver L. Aspectos metodológicos em avaliação dos serviços de saúde. In: Gallo E, Rivera FJU, Machado $\mathrm{MH}$, organizadores. Planejamento criativo: novos desafios em políticas de saúde. Rio de Janeiro: Editora Relume-Dumará; 1992. p. 195-209.

6. Paim J. Avaliação em saúde: uma prática em construção no Brasil. In: Hartz ZM, Vieira-da-Silva LM, organizadoras. Avaliação em saúde: dos modelos teóricos à prática na avaliação de programas e sistemas de saúde. Rio de Janeiro: Editora Fiocruz; 2005. p. 9-11.

7. Guba EG, Lincoln YS. Fourth generation evaluation. Newbury Park: Sage Publications; 1989. 
8. Stake RE. Evaluación compreensiva y evaluación basada em estándares. Barcelona: Editorial Graó; 2006.

9. Shadish WR, Cook TD, Leviton LC, editors. Foundations of program evaluation: theories of practice. Newbury Park: Sage Publications; 1991.

10. Rossi PH, Lipsey MH, Freeman HE. Evaluation: a systematic approach. Thousand Oaks: Sage Publications; 2004.

11. Cahiers Internationaux de Sociologie 2010; CXXVIII-CXXIX:1-192.

12. Cités, Philosophie, Politique, Histoire 2009; 37:1211.

13. Bourdieu P. As regras da arte. São Paulo: Editora Companhia das Letras; 2010.

14. Bourdieu P. O senso prático. Petrópolis: Editora Vozes; 2009.

15. Bourdieu P, Wacquant L. Réponses. Paris: Seuil; 2000.

16. World Bank. Towards the institutionalization of monitoring and evaluation systems in Latin America and the Caribbean. Washington DC: World Bank; 2006.

17. World Bank. Health sector project: Reforsus. Washington DC: World Bank; 1996. (Staff Appraisal Report, 15522-BR).

18. Santos GF. Investimentos e reforma do setor saúde no Brasil: o caso do projeto Reforsus. In: Bayama F, Kasznar I, organizadores. Saúde e previdência social: desafios para o terceiro milênio. São Paulo: Editora Pearson Education; 2003. p. 227-32.

19. Alves GJW. Equidade e os projetos de investimento em saúde: possibilidades e limites do projeto Reforsus [Dissertação de Mestrado]. Brasília: Departamento de Serviço Social, Universidade de Brasília; 2009

20. Viana ALd'A, Dal Poz MR. A reforma do sistema de saúde no Brasil e o Programa de Saúde da Família. Physis (Rio J.) 2005; 15:225-64.

21. Felisberto E. Da teoria à formulação de uma Política Nacional de Avaliação em Saúde: reabrindo o debate. Ciênc Saúde Coletiva 2006; 11:553-63.

22. Hartz ZMA, Felisberto E, Vieira-da-Silva LM. Meta-avaliação da atenção à saúde: teoria e prática. Rio de Janeiro: Editora Fiocruz; 2008.

23. Campos FCB, Henriques CMP, organizadores. Contra a maré à beira-mar: a experiência do SUS em Santos. São Paulo: Editora Hucitec; 1997.
24. Facchini LA, Costa J. Utilização de serviços ambulatoriais em Pelotas: onde a população consulta e com que frequência. Rev Saúde Pública 1997; 31:360-9.

25. Vieira-da-Silva LMA. Descentralização das ações de saúde no município: o caso de Camaçari [Tese de Doutorado]. São Paulo: Faculdade de Medicina, Universidade de São Paulo; 1990.

26. Fernandes FMB, Ribeiro JM, Moreira MR. Reflexões sobre avaliação de políticas de saúde no Brasil. Cad Saúde Pública 2011; 27:1667-77.

27. Colussi CF, Calvo MCM. Avaliação da atenção em Saúde Bucal no Brasil: uma revisão da literatura. Saúde \& Transformação Social 2012; 3:92-100.

28. Canella DS, Silva ACF, Jaime PC. Produção científica sobre nutrição no âmbito da atenção primária à saúde no Brasil: uma revisão de literatura. Ciênc Saúde Coletiva 2013; 18:297-308.

29. Almeida PF, Giovanella L. Avaliação em Atenção Básica à Saúde no Brasil: mapeamento e análise das pesquisas realizadas e/ou financiadas pelo Ministério da Saúde entre os anos de 2000 a 2006. Cad Saúde Pública 2008; 24:1727-42.

30. Akerman M, Nadanovsky P. Avaliação dos serviços de saúde: avaliar o quê? Cad Saúde Pública 1992; 8:361-5.

31. Minayo MCS. Abordagem antropológica para avaliação de políticas sociais. Rev Saúde Pública 1991; 25:233-8.

32. Silva LMV, Formigli VLA. Avaliação em saúde: limites e perspectivas. Cad Saúde Pública 1994; 10: 80-91.

33. Donabedian, A. Explorations in quality assessment and monitoring. Ann Arbor: Health Administration Press; 1980.

34. Hartz ZMA, organizadora. Avaliação em saúde: dos modelos conceituais à prática na análise da implantação de programas. Rio de Janeiro: Editora Fiocruz; 1997.

35. Ciênc Saúde Coletiva 2003; 11:550-850.

36. Vieira-da-Silva LM, Pinell P. The genesis of collective health in Brazil. Sociol Health Illn 2014; 36:432-46.

37. Bourdieu P. Homo academicus. Florianópolis: Editora UFSC; 2011.

Recebido em 31/Out/2013

Versão final reapresentada em 06/Mai/2014

Aprovado em 29/Mai/2014 